\title{
Android Based Defence Robot
}

\author{
Punitha A, Raghupathi S
}

\begin{abstract}
Being in the era where the defense robots are implemented, for the purpose of reconnaissance, breaching missions were the patrols have never tried in the war field. In previous conventional systems, the terrain infantry's tough jobs had been done by utilizing zig-bee based teleoperation systems, CMOS cameras \& LCD displays. The proposed system is improved with computerized display and Android-based ethical hacking technique. This is to achieve a live video streaming with less time and power consumption between the peripherals. The newly proffered system involves a bot that can perform all directional movements, night vision, pick and place by using gripper, gun projection control. It is based on the user's instruction from the control room the robot can perform laser shoot and sensing temperature conditions. This can be done simply by utilizing Node MCU (ESP 8266(Wi-Fi module) with inbuilt microcontroller), Android mobile phone, servo motors and a DHT22 or DHT11 (temperature sensor).
\end{abstract}

Key words- Node MCU, Servo motors, DC motors, Blynk app, IP web cam, Ethical Hacking, DHT 11, Arduino IDE.

\section{INTRODUCTION}

The primary goal of the paper is to secure the precious life of the patrols and to reduce the causality of the terrorist. The enhancement of the defense robot is that it can be able to perform multitasking operations through Wireless Fidelity (Wi-Fi). Similarly, the directional movements, the pick and place movements and gun control with laser shoot operations can be performed by the person from remote areas.

\section{EXISTING SYSTEM}

The conventional robots were implemented with certain features like controlling all possible directional movements, temperature sensing, pick and place along with gun control movements by utilizing different terminologies. Those terminologies utilized microcontrollers such as raspberry pi, Web Input Output PI (WEBIOPI), different Robot Operating System (ROS) and Peripheral Interface Controller (PIC) boards. Blue-tooth, WI -FI and high power Zig- bee were used for the transmission of data from a controller region to the reception areas. The Robots have been controlled by utilizing Nintendo Wifi remote and Third Generation (3G) technology for video transmission. Android studio environment is used for writing Java program codes in order to feed the robot and virtual reality technologies. Some application software has been

Revised Manuscript Received on November 22, 2019

A.Punitha,Associate Professor Department Electronics and Communication Engineering Sudharsan College of Engineering, Pudukkottai.

S.Raghupathi, Lecturer Department of Engineering, IBRI College of Technology, Oman. utilized based on Internet Of Things (IOT) for connecting and sharing the data between the Access Points (AP).

\section{PROPOSED SYSTEM}

The proposed system has developed with a few techniques such as the computerized display for live video streaming and updating current weather conditions. The robot can able to perform bi- directional movements which can be achieved by using DC motors. The gripper control movement allows it to make a necessary response to the commands based on pick and place movements. The gun controlling mechanism along with laser shoot can be performed by the instruction that has been transmitted from the control room. The atmospheric temperature and weather conditions of the robot will be predicted by using a temperature sensor, which converts the observed analog data into the digitized form and will be provided in the computerized display. This robot has a feature night vision for the mission to be accomplished even at the night times by operating the LEDs attached with it. The hardware part includes Node MCU as a Wi-Fi module with an inbuilt microcontroller which performs wireless transmissions with less cost by reducing the delay between the transmitting and receiving end. DHT11 or DHT22 has been attached with the microcontroller in order to observe the weather conditions of the field where the robot is placed. LEDs are provided to get enough Light vision at situations when the robot is in need to perform surveillance by means of darkness. The space of software tools is occupied by utilizing Arduino IDE software for coding and feeding that particular code into ESp8266- Node MCU. This software developing environment is found to be very adaptive and user- friendly from the developer side. It enables the developers to build their codes with the available open sources and implementing it through hardware to form an embedded environment. Fig .1 and Fig. 2 explains about the implementation of the robot using the hard ware components.

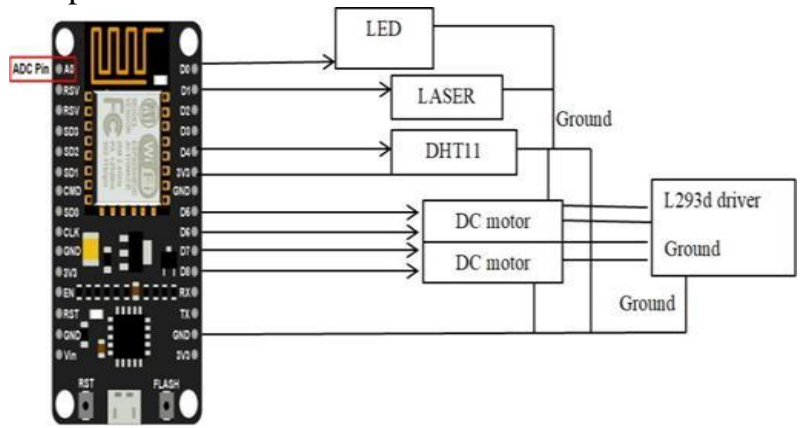

Fig.1. Circuit diagram for implementing the directional movements with laser shoot and current updates of temperature conditions.

Blue Eyes Intelligence Engineering 


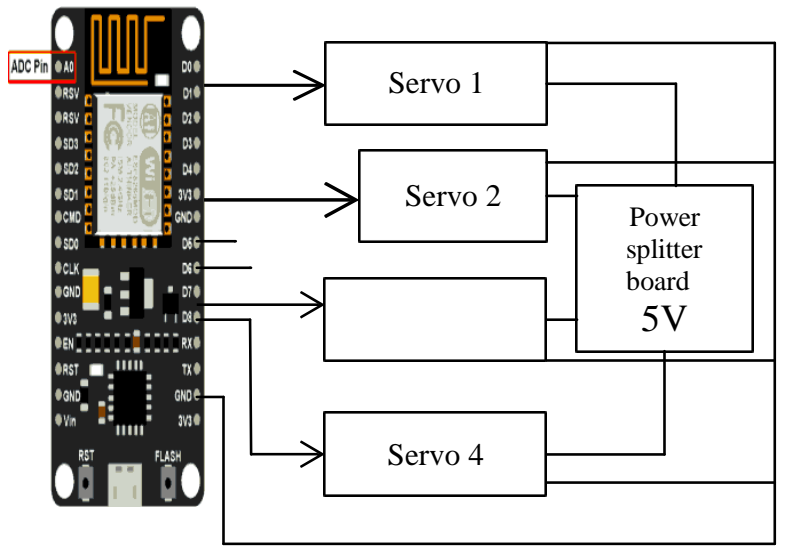

Fig. 2. Circuit diagram for implementing the pick and place movements, Surveillance and gun control movements by using servo motors.

\section{WORKING PROCEDURE}

The General block diagram of Android based defense robot with all the application is shown in the Fig. 3.

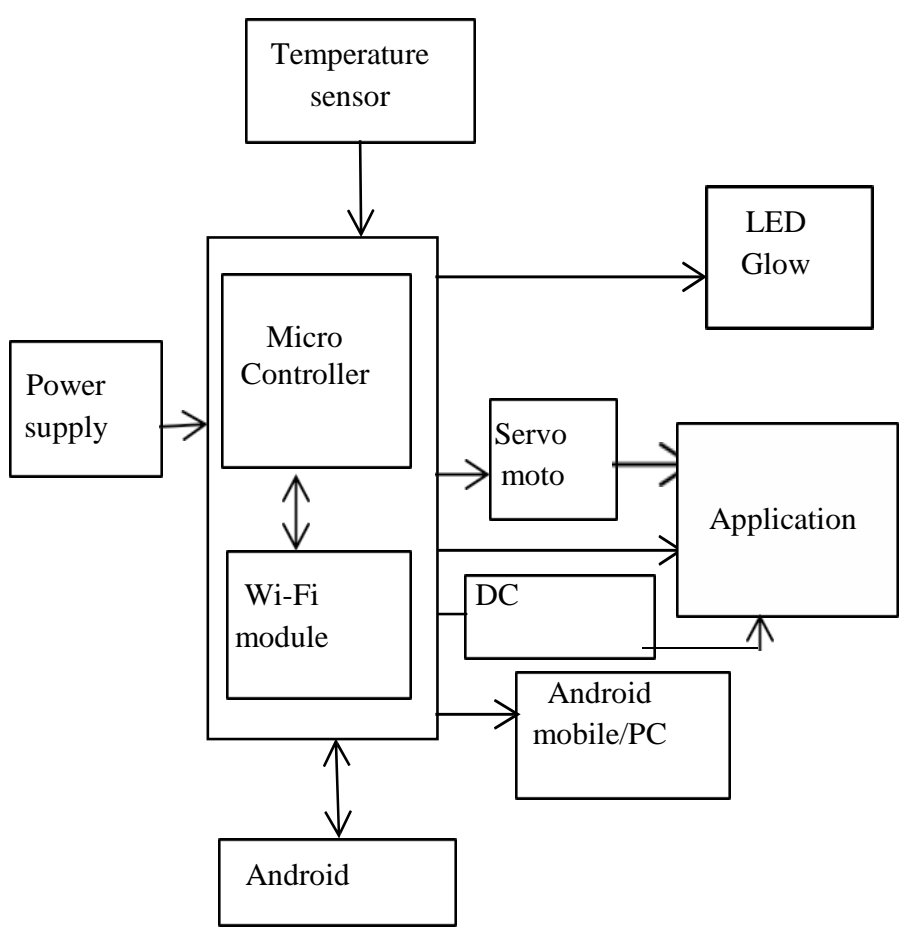

Fig.3. General block diagram of the Android based defense robot.

The system consists of three main division, they are 1.Control unit 2.Monitoring the robot and 3.Live streaming through ethical hacking. The control unit is provided with either a Personal Computer (PC) or an android mobile phone where the robot's action can be controlled through a web page filled with icons and widgets. The command that get triggered from the control room can be executed by performing necessary actions as per the program is designed. Monitoring the robot is to look after the actions performed by the robot at every instance and situations. The live video coverage from the war field can be done through ethical hacking technique, in which two devices get connected with necessary authentications and ensuring highly secured transmission of data. Since, the data which

gets propagated from one device to another is found to be encrypted provides an assurance for complete security and privacy.

The controlling operations can be done through Blynk community that comes under the Android platter which enables to create widgets to control the robots utilizing online environment. Since a single Node MCU cannot uptake all the features that are involved in the applications of the robot. It can be divided into two sections as already shown in the circuit diagrams that are involved in the Fig. 1. and Fig. 2. Hence two set of widgets were created by using Blynk application were one set is to control the servo motors which are in account to perform the surveillance operation, gun control and pick and place movements. Where the other widget enable to control the directional movements, Laser shoot operation, night vision light and current updates of temperature condition through DHT11 temperature sensor.

The code can be generated by using the Blynk code generator from the web browser. It is an online platform provides the necessary open source that enables the developers to create a code that deals with the implementation of the project. The code can be developed through analyzing the authentication ID in which the Blynk application is reqistered. Fig. 4. and Fig. 5. shows the widgets that are created for controlling the entire application of the robot by using Blynk application.

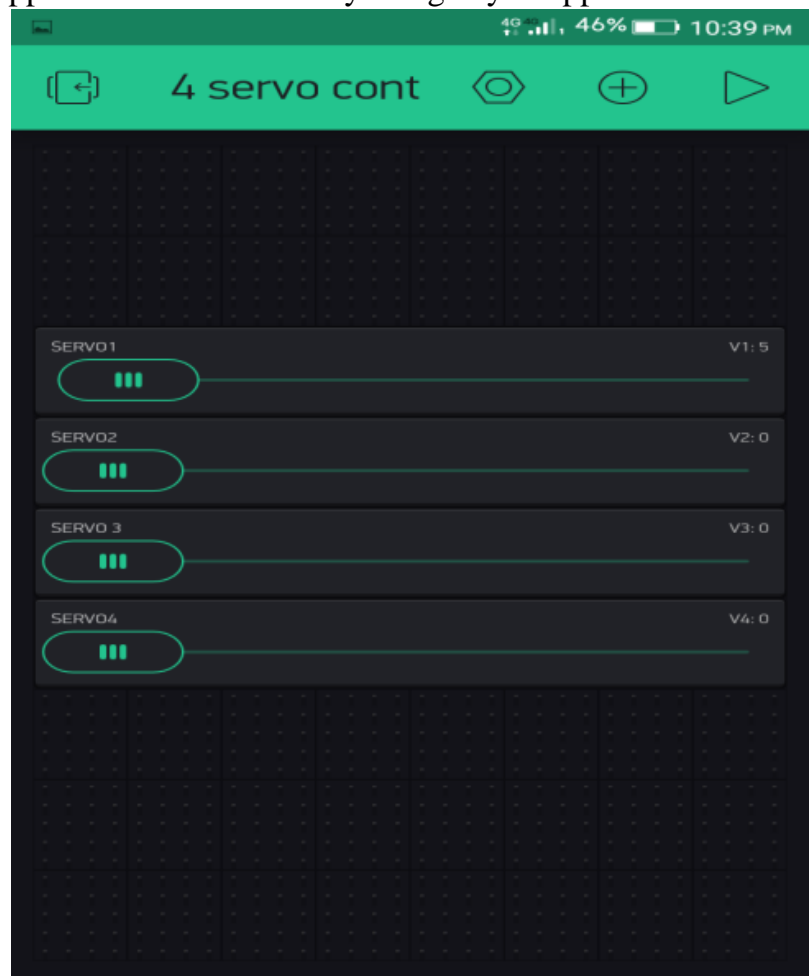

Fig.4. Widget created to control the pick and place movements, gun control movements and surveillance using Blynk.

Published By: 


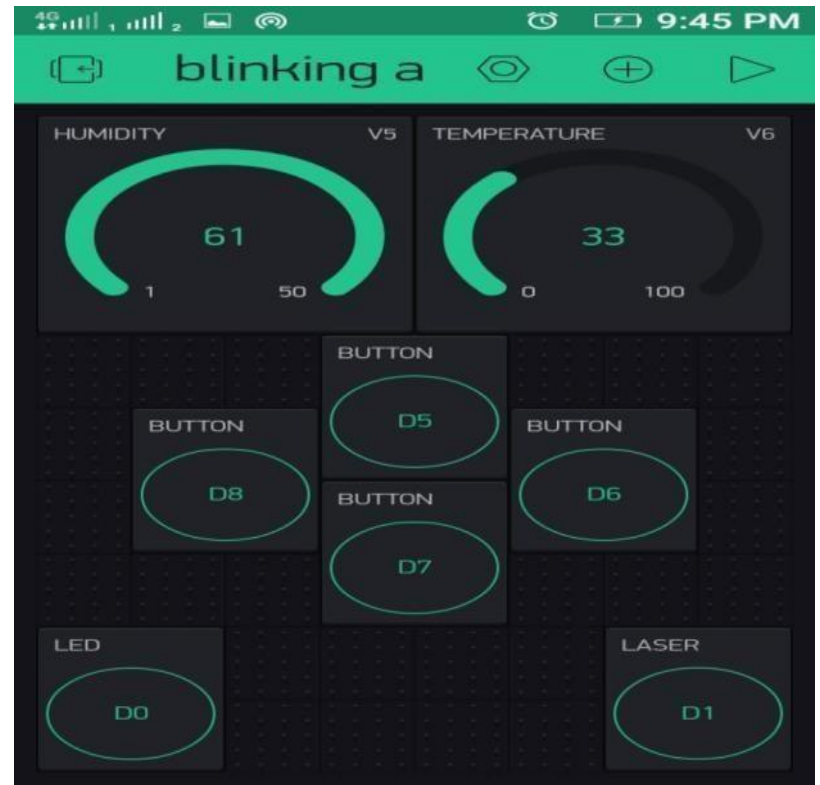

Fig.5. Widget created for controlling Laser shoot,

Directional movements, temperature and humidity updates and night vision light by using Blynk.

The surveillance application can be done through the Android mobile by providing the Internet Protocol (IP) web cam which gets connected through Transmission Control Protocol (TCP/IP) over the internet layer between the peripherals.

This provides live video streaming of data by ensuring privacy and security throughout the propagation time. The generated IP address is entered into the browser which enables the streaming of video with complete audio coverage and less delay in transmission.

The development of code for feeding into the Node MCU which is a wi -fi module with inbuilt micro controller enables a large spectral width and less time delay for transmission. The module has to be feed with the developed code using Arduino Integrated development Environment (IDE). The Blynk library must be installed and added along with the code by using the board manager option which is provided by the IDE.

The live video streaming of data and visualized through a Personnel computer (PC) is illustrated in Fig.6.

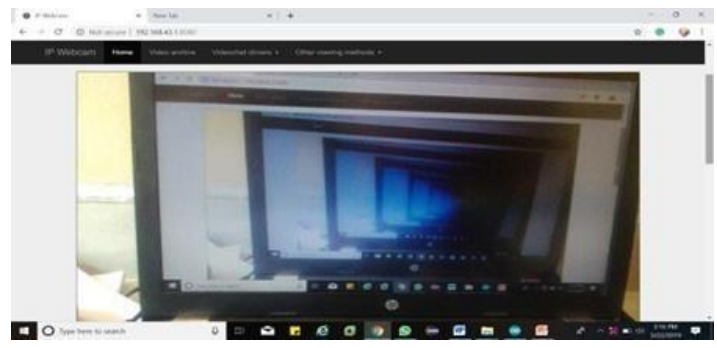

Fig. 6. Live video streaming of IP web cam application through web browser and visualized by using a PC.

\section{CONCLUSION}

This Android based defense robot in the patrol application must be utilized by the people who are well trained and tested with the basic knowledge. The patrols must be provided with the necessary skill of rectifying the technical and mechanical causes which arises while involved in performing certain applications. It will be better to provide necessary environment to develop any other additional features that are in need to be included with the bot to perform any other specialized applications. Because the system is provided with quality features that can provide enough support for the future development and attachments. The system can be enhanced by expanding spectral width and the area of coverage based on the improvement of the future technologies. The future works includes mainly enhancing the quality of camera with high pixel resolution instead of utilizing an ordinary inbuilt camera in an Android mobile. The patrols and infantries must be trained to utilize the robot before they enter into the war field. The delay for transmitting the data can also be reduced so that it may reduce the tough situations that are faced by the people in the war field. Utilization of IR sensors and RFID can be applied for the purpose of recognizing the native members who utilize the Robot.

\section{REFERENCES}

1. Aathish Bankar, Anuja Bhilare,Namrata singh, Kunal Ranvir, (March 2016), "Defense Robot With Gun Assembly" International Engineering Research Journal (IERJ) ISSN 2395-1621, 2(1), Page 376-378.

2. Berkay Gokcen, Fatih Baygul, Furkan Cakmak, Erkan Uslu, M.Fatih Amasyali, Sirma Yavuz,(Oct 2017), "Android application for simultaneously control of multiple land robots which have different drive strategy" in the International conference on Computer Science and Engineering. Available at DOI: 10.1109/UBMK.2017.8093513

3. Chinnaiah M C,(Mar 2015), "FPGA based robots hardware efficient scheme for Real-time indoor environment with behavioral control" in the International Conference on Innovations in Information, Embedded and Communication Systems (ICIIECS).

4. Christos Papachristos, Dimos Tzoumanikas, Anthony Tzes, (Dec 2015), "Aerial robotic tracking of a generalized mobile target employing visual and spatiotemporal dynamic subject perception", in the International Conference on Intelligent Robots and Systems (IROS). Available at DOI: 10.1109/IROS.2015.7353989

5. Dhanalakshmi.V, Jayasri.D, Vishnupriya.R, (April 2014),"Zigbee Based Wireless Intelligent Security Robot For Defence Camp", International Journal of Advanced Engineering and Global Technology(IJAEGT), ISSN No: 2309-4893, 2 (4).

6. Mandar Kulkarni, Abhishek Mishra, Pranjal Barabde, Dhvani Shah, (Mar 2017), "IOT Based Multifunctional ROBOT using RASPERRY-PI" International Journal of Advances in Electronics and Computer Science (IJAEC), ISSN: 2393- 2835,4(3).

7. Navaneetha Krishna Kumar.S, R. J. Praveen Kumar, M. Raja Kumaran, G. Valarmathy, (Mar 2016), "Design and control of defense robot based on virtual reality" International Journal of Science and Research (IJSR),5(3).

8. Sahil Bhatnagar, Shivam Kumar Gola, Pooja Agarwal, Shivam Bhatia, Pragati Gupta, (Oct 2016), "A review on rough terrain and defense robot" International Journal of scientific research and management (IJSRM),4(10).

9. Sweeta Deshmukh, Priyadarshini, Mamta, Madhura Deshmukh, Md.Bakhar, (June 2017), "IOT Based Surveillance Robot" International Journal of Innovative Research in Computer and Communication Engineering (IRCCE). An ISO 3297: 2007 Certified Organization $2^{\text {nd }}$ National Conference on Recent Advances in Engineering and Technology (NCRAET_2017) 5(4).

10. Tarunpreet Kaur, Dilip Kumar, (Dec 2015), "Wireless multifunctional robot for military aplications", in the International Conference on Recent Advances in Engineering \& Computational Sciences (RAECS). 


\section{Android Based Defence Robot}

\section{AUTHORS PROFILE}

A.Punitha, Currently, (Arockiasamy Punitha) working as Associate Professor in the Department Electronics and Communication Engineering at Sudharsan College of

Engineering, Pudukkottai. She acquired her Bachelor's degree in Electronics and Communication Engineering from Bharathidasan University, Tiruchirapalli in 2002. She obtained her Master's degree in Communication Engineering from Anna University, Chennai in 2009 and Ph.D degree from Anna University, Chennai. She has over 15 years of experience in teaching and guiding projects. Her areas of interests include Vehicular Ad hoc Networks, Wireless Sensor Networks, Digital Communication, Optical Communication and Network Security.

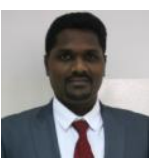

S.Raghupathi (Senthilvel Raghupathi) obtained his Bachelor's degree in Electronics and Communication Engineering from Bharathidasan University, Tiruchirapalli in 2002. Then he obtained his Master degree in VLSI

Design from SASTRA University, Tanjore. Currently, he is serving as a Lecturer in Department of Engineering, at IBRI College of Technology,Oman. His specializations include VLSI design, Communication Engineering, and networking. His current research interests are Bluetooth Scatter net Formation, Public Key Infrastructure, Network Security, and Authentication Server and VANET's. 